\title{
Multi-step synthesis of nanoparticles performed on millisecond time scale in a microfluidic droplet-based system $\dagger$
}

\author{
Ilya Shestopalov, Joshua D. Tice and Rustem F. Ismagilov* \\ Department of Chemistry, The University of Chicago, 5735 South Ellis Avenue, Chicago, IL \\ 60637, USA. E-mail: r-ismagilov@uchicago.edu
}

Received 4th March 2004, Accepted 12th May 2004

First published as an Advance Article on the web 5th July 2004

\begin{abstract}
This paper reports a plug-based, microfluidic method for performing multi-step chemical reactions with millisecond time-control. It builds upon a previously reported method where aqueous reagents were injected into a flow of immiscible fluid (fluorocarbons) (H. Song et al., Angew. Chem. Int. Ed., 2003, 42, 768). The aqueous reagents formed plugs - droplets surrounded and transported by the immiscible fluid. Winding channels rapidly mixed the reagents in droplets. This paper shows that further stages of the reaction could be initiated by flowing additional reagent streams directly into the droplets of initial reaction mixture. The conditions necessary for an aqueous stream to merge with aqueous droplets were characterized. The Capillary number could be used to predict the behavior of the two-phase flow at the merging junction. By transporting solid reaction products in droplets, the products were kept from aggregating on the walls of the microchannels. To demonstrate the utility of this microfluidic method it was used to synthesize colloidal $\mathrm{CdS}$ and CdS/CdSe core-shell nanoparticles.
\end{abstract}

\section{Introduction}

This paper describes a droplet-based ${ }^{1}$ microfluidic method for performing multi-step synthesis of nanoparticles on millisecond time scale. The utility of this method was demonstrated by synthesizing $\mathrm{CdS}$ and $\mathrm{CdS} / \mathrm{CdSe}$ nanoparticles in two steps in aqueous solution. Microfluidics is a rapidly growing area that is interesting both scientifically and technologically. ${ }^{2-6}$ Microfluidic networks have been shown to be useful in performing synthetic reactions ${ }^{7-9}$ because fluid flow through microchannels has the potential to relate reaction time to the distance that reagents have traveled through channels. Multiphase flow in microfluidic devices has been used for DNA analysis, ${ }^{10}$ nitration ${ }^{11}$ and fluorination ${ }^{12}$ of aromatics, catalytic hydrogenation of alkenes, ${ }^{13}$ and acid/base titrations. ${ }^{14}$ When performing reactions in microfluidic channels, ideally, one could relate distance to time by the simple formula $t=$ $d / U$ where $t[\mathrm{~s}]$ is elapsed time of the reaction, $d[\mathrm{~m}]$ is the distance traveled through a channel, and $U\left[\mathrm{~m} \mathrm{~s}^{-1}\right]$ is the flow velocity. One encounters two problems when trying to achieve millisecond timecontrol in microfluidic reaction networks. First, mixing of two laminar reagent streams at low Reynolds number in microfluidic channels proceeds by diffusion and is slow $(R e=\rho U l / \mu$ where $\rho$ $\left[\mathrm{kg} \mathrm{m}^{-3}\right]$ is the density of the fluid, $l[\mathrm{~m}]$ is a characteristic length scale of the channel-the cross-sectional dimension, and $\mu\left[\mathrm{kg} \mathrm{m}^{-1}\right.$ $\mathrm{s}^{-1}$ ] is the viscosity of the fluid). ${ }^{15-17}$ Slow mixing introduces a high degree of uncertainty to the starting time of the reaction. ${ }^{1}$ Second, reagents are dispersed along the channel in a laminar flow because of a parabolic flow profile that results from no slip boundary conditions at the walls. ${ }^{16}$ Turbulent flow at high $R e$ mixes reagents more quickly and limits dispersion, but requires high flow rates and increased sample consumption. ${ }^{18}$

We have previously demonstrated millisecond time control of a single-step reaction in microfluidic channels by isolating multiple streams of reagents within plugs - aqueous droplets surrounded and transported by fluorocarbons immiscible with water. ${ }^{1}$ We refer to the fluorocarbon mixtures used in this study as "oils". Transport of reaction mixtures within droplets eliminates dispersion. Reagents isolated within droplets flowing though winding channels can be mixed on sub-millisecond time scale by chaotic advection. ${ }^{19} \mathrm{We}$ have used this droplet-based microfluidic method to perform rapid

$\dagger$ Lab on a Chip special issue: The Science and Application of Droplets in Microfluidic Devices. kinetics measurements of single stage reactions ${ }^{20}$ and to perform protein crystallizations. ${ }^{21,22}$ Several methods of forming droplets in microfluidic channels have been described, and gas bubbles have also been used to enhance the mixing of liquids. ${ }^{1,23-26}$

To perform multi-step, time controlled syntheses with this droplet-based microfluidic method, additional reagent must be added to the droplets formed from initial reagents. Previously, we accomplished this addition by merging one stream of droplets with a second stream of droplets. ${ }^{1}$ This method allowed two separate reactions to be performed in parallel and then combined. This method worked well, but special measures had to be taken to assure that the two streams of droplets were brought together in a synchronized fashion. The frequency of droplets entering a main channel from one of the peripheral channels needed to be identical to the frequency of droplets entering from a second peripheral channel. One set of droplets needed to be larger than the second set, and an additional stream of water-immiscible fluorinated fluid was needed to make the second stream of plugs.

Here, we report an alternative approach to adding reagents to droplets - direct injection of aqueous reagent into flowing droplets. We also report that droplets may be used to encapsulate solid reaction products and prevent them from contacting the walls of microchannels. As a demonstration of both of these aspects of droplet-based synthesis method, we synthesized colloidal CdS nanoparticles in a two-step process, controlled on millisecond time scale.

\section{Experimental}

We fabricated microfluidic devices out of polydimethylsiloxane (PDMS), infused fluids, and took micrographs as previously reported. ${ }^{25}$ Microfluidic channels were approximately $50 \mu \mathrm{m}$ high, $50 \mu \mathrm{m}$ wide, and $24.5 \mathrm{~mm}$ long from droplet-forming region to quenching region. The colorless aqueous streams in Figs. 1, 2, and 3 were solutions of $\mathrm{KNO}_{3}(0.1 \mathrm{M})$. Colored aqueous streams were solutions of $\mathrm{Fe}(\mathrm{SCN})_{x}(3-x)+(0.03 \mathrm{M})$ in $\mathrm{KNO}_{3}(0.1 \mathrm{M})$. Waterimmiscible fluorinated oil was a mixture of $3 \mathrm{M}$ Fluorinert fluid FC72/ 3M Fluorinert fluid FC-84/ 1H,1H,2H,2H-perfluoro-1-octanol $(50: 50: 1 \mathrm{w} / \mathrm{w} / \mathrm{w})$. In the experiment performed with viscous solutions, colorless aqueous streams were solutions of $\mathrm{KNO}_{3}(0.1$ $\mathrm{M})$ in $68 \%$ glycerol in water $(\mu=15.6 \mathrm{mPa} \mathrm{s})$. Colored aqueous streams were solutions of $\mathrm{Fe}(\mathrm{SCN})_{x}{ }^{(3-x)+}(0.03 \mathrm{M})$ in $68 \%$ glycerol solution of $\mathrm{KNO}_{3}(0.1 \mathrm{M})$. Water-immiscible fluorinated oil was a mixture of perfluoroperhydrophenanthrene/ 


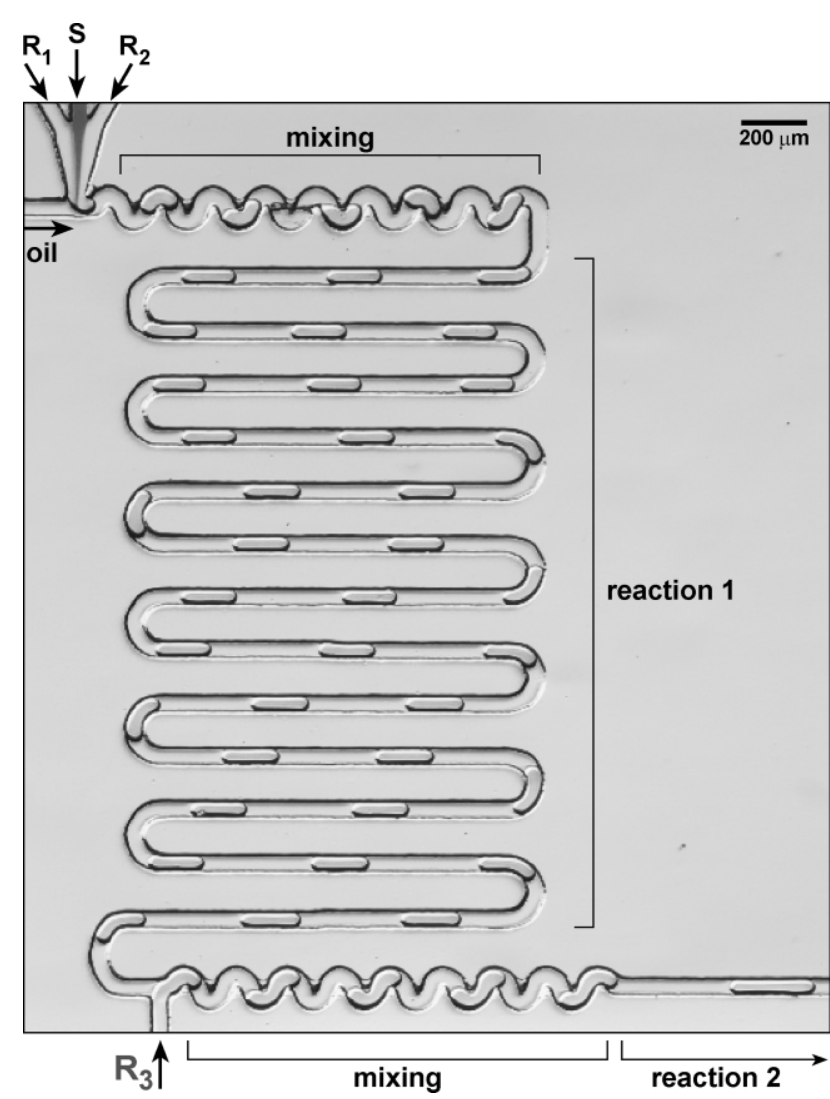

Fig. 1 A micrograph of a microfluidic device for performing dropletbased, two-step synthesis with millisecond time control. Aqueous reagents $\mathrm{R}_{1}, \mathrm{R}_{2}$, and a separating stream $\mathrm{S}$ form droplets of the initial reaction mixture. Winding channels induce rapid mixing in droplets and initiate reaction 1 . Reaction 2 is initiated when the stream of aqueous reagent $R_{3}$ adds to aqueous droplets of the initial reaction mixture and the resulting droplets are mixed. Flow velocity was $U=67 \mathrm{~mm} \mathrm{~s}^{-1}$, and $w f=0.4$. Water fraction, $w f$, is defined in the text.

(a)

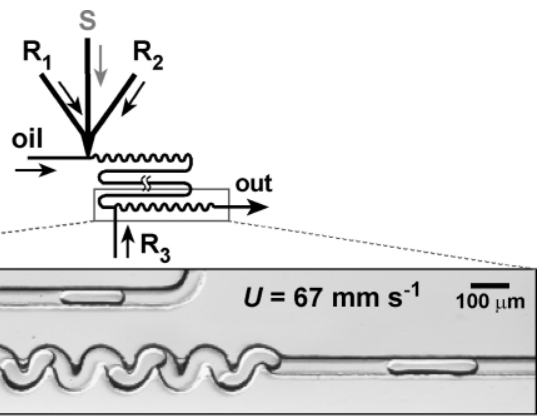

(b)
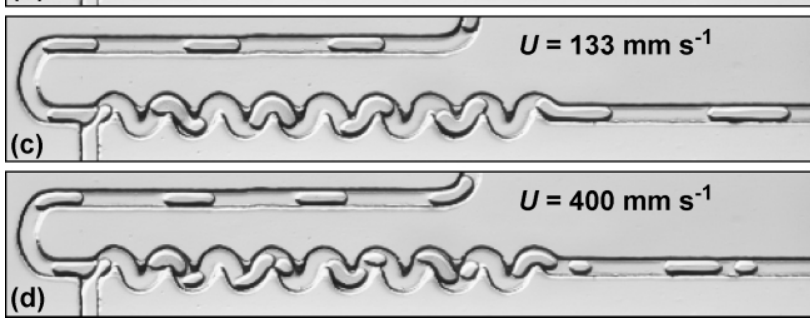

Fig. 2 Merging of an aqueous stream with aqueous droplets as a function of the flow velocities of the droplets and the stream. (a) A schematic diagram of the microfluidic network. (b) Micrograph showing aqueous droplets merging immediately with the aqueous stream $\left(U=67 \mathrm{~mm} \mathrm{~s}^{-1}\right)$. (c) Micrograph showing aqueous droplets that merge with a droplet of the additional aqueous stream $R_{3}$ shortly after flowing past the junction $(U=$ $133 \mathrm{~mm} \mathrm{~s}^{-1}$ ). (d) Microphotograph showing aqueous droplets not merging with the aqueous stream $\left(U=400 \mathrm{~mm} \mathrm{~s}^{-1}\right)$. The aqueous stream forms separate droplets. In all micrographs, $w f=0.4$.
1H,1H,2H,2H-perfluoro-1-octanol (100:1 w/w). Prior to experiments, a mixture of $3 \mathrm{M}$ Fluorinert fluid $\mathrm{FC}-84 / 1 \mathrm{H}, 1 \mathrm{H}, 2 \mathrm{H}, 2 \mathrm{H}-$ perfluoro-1-octanol $(20: 1 \mathrm{w} / \mathrm{w})$ was infused into the channels at 15

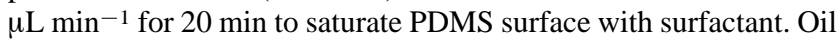
to be used in the experiment was then pumped through at $10 \mu \mathrm{L}$ $\min ^{-1}$ for $20 \mathrm{~min}$ to remove excess surfactant.

All nanoparticle syntheses were performed in Millipore filtered $\mathrm{H}_{2} \mathrm{O}$ degassed with $\mathrm{N}_{2}$. Synthesis of CdS/CdSe nanoparticles was done under $\mathrm{N}_{2}$ atmosphere. All solutions were used within $3 \mathrm{~h}$ of preparation. 1-Mercaptopropionic acid (MPA), $\mathrm{CdCl}_{2}$, and $\mathrm{Na}_{2} \mathrm{~S}$, were obtained from Aldrich. $\mathrm{Na}_{2} \mathrm{Se}$ was obtained from Alfa Aesar. All reagents were used without further purification. In a typical preparation $\mathrm{CdCl}_{2}(0.8 \mathrm{mM})$ was initially premixed with MPA $(0.8$ $\mathrm{mM}$ ) and a solution of $\mathrm{NaOH}$ was used to bring the mixture to $\mathrm{pH}$ 11. This solution of $\mathrm{CdCl}_{2} / \mathrm{MPA}$ and solutions of $\mathrm{Na}_{2} \mathrm{~S}(0.8 \mathrm{mM})$, $\mathrm{Na}_{2} \mathrm{Se}(0.8 \mathrm{mM}), \mathrm{NaOH}$ (pH 11), and MPA quench (60 mM, pH 11) were infused into the microfluidic device at $8 \mu \mathrm{L} \mathrm{min}-1$. Oil was infused at $25 \mu \mathrm{L} \mathrm{min}-1$. Agitation of the microfluidic device was performed mechanically at $10 \mathrm{~Hz}$. In the 20 -fold excess of $\mathrm{CdCl}_{2} /$ MPA experiment, $\mathrm{CdCl}_{2}(4 \mathrm{mM})$ was initially premixed with MPA $(5 \mathrm{mM})$ and a solution of $\mathrm{NaOH}$ was used to bring the mixture to $\mathrm{pH}$ 11. This mixture of $\mathrm{CdCl}_{2} / \mathrm{MPA}$ was infused at $16 \mu \mathrm{L} \mathrm{min}-1$, while solutions of $\mathrm{Na}_{2} \mathrm{~S}(0.8 \mathrm{mM})$, and $\mathrm{NaOH}(\mathrm{pH} 11)$ were infused at 4 $\mu \mathrm{L} \mathrm{min}{ }^{-1}$. Excess MPA was filtered off from the collected sample through a Millipore Ultrafree 0.5 centrifugal filter device. In the benchtop experiment, a mixture of $\mathrm{CdCl}_{2} / \mathrm{MPA}(0.5 \mathrm{~mL}, 0.8 \mathrm{mM}$, $\mathrm{pH} 11)$ and solutions of $\mathrm{NaOH}(0.5 \mathrm{~mL}, \mathrm{pH} 11), \mathrm{Na}_{2} \mathrm{~S}(0.5 \mathrm{~mL}, 0.8$ $\mathrm{mM})$, and MPA $(0.5 \mathrm{~mL}, 60 \mathrm{mM}, \mathrm{pH} \mathrm{11})$ were combined in that order under vigorous stirring. Optical absorption spectra were acquired using a Hewlett-Packard 8453 diode array spectrometer. Photoluminescence spectra were acquired using SPEX Fluoromax3 fluorimeter.

\section{Results and discussion}

In this microfluidic method to multi-step synthesis on millisecond time scale, we first formed droplets of the initial reaction mixture (Fig. 1). Two aqueous reagent streams were brought together in a short segment of channel where they were allowed to flow laminar alongside each other (colorless streams labeled $R_{1}$ and $R_{2}$ in Fig. 1). These reagent streams were separated by an inert stream (labeled $S$ and dyed red in Fig. 1). The separating stream prevented reagents from mixing ${ }^{15-17}$ until they entered a flow of water-immiscible,

(a)
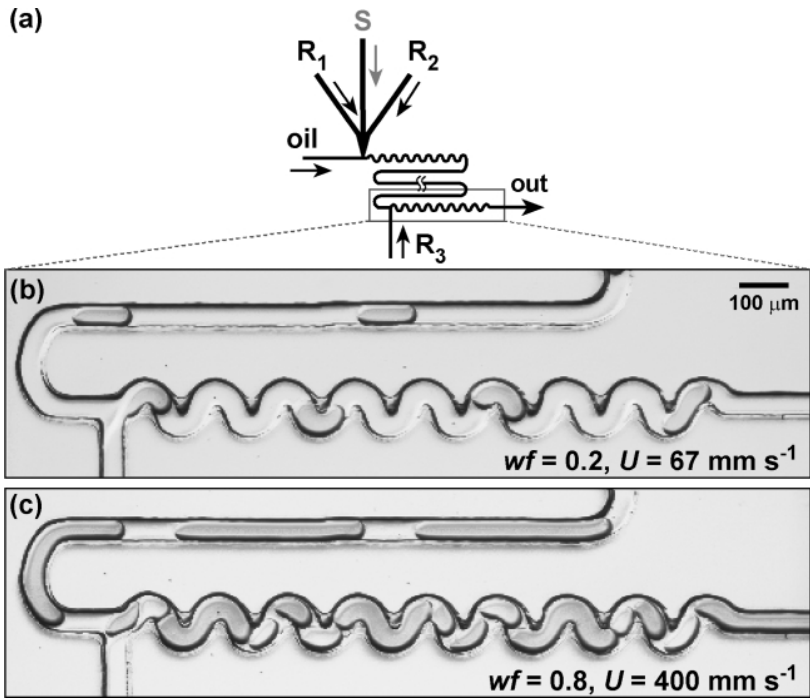

Fig. 3 At all water fractions, conditions for merging depend on flow velocities. (a) A schematic diagram of the microfluidic network. (b) A micrograph showing droplets merging with the aqueous stream at $w f=0.2$, $U=67 \mathrm{~mm} \mathrm{~s}^{-1}$. (c) A micrograph showing droplets not merging with the aqueous stream at $w f=0.8, U=400 \mathrm{~mm} \mathrm{~s}^{-1}$. Shading on the upper side of the droplets is an artifact of non-uniform lighting and contrast enhancement. 
fluorinated oil where the aqueous streams spontaneously broke up into droplets surrounded and separated by the oil. We refer to these droplets of initial reaction mixture as the initial droplets. Winding channels induced rapid mixing by chaotic advection. ${ }^{1,19}$ Submillisecond mixing times can be achieved inside droplets moving at high velocities through channels of small cross-sectional dimensions, ${ }^{19}$ and mixing times of less than $2 \mathrm{~ms}$ have been obtained in droplets in channels similar to the ones shown in Fig. 1 at flow velocities of $300 \mathrm{~mm} \mathrm{~s}^{-1} .^{1}$ Therefore, the short segment of winding channel labeled "mixing" in Fig. 1 is adequate to mix aqueous solutions at any flow velocity. After mixing, the droplets were allowed to react while flowing through a serpentine section of the channel (labeled "reaction 1" in Fig. 1). Because a distance-to-time relationship is preserved in this microfluidic method, reaction times could be controlled by adjusting flow rates or by designing and fabricating networks with different lengths of channel between junctions. Reaction time could be as short as $5 \mathrm{~ms}$ or less, and as long as $\sim 1 \mathrm{~min}$. To initiate the second stage of the multi-step reaction, an aqueous stream of an additional reagent (colorless stream labeled $R_{3}$ in Fig. 1) was directly injected into the droplets at a junction referred to as the merging junction. The resulting droplets were mixed as they flowed through a winding section of channel, and the second reaction proceeded as droplets flowed through another length of serpentine or straight channel. Similarly, additional stages could be initiated further downstream. The amount of reagent added to droplets could be adjusted by changing the flow rate of the additional reagent streams.

We characterized the conditions under which an aqueous stream, $\mathrm{R}_{3}$, would reliably inject into a moving aqueous droplet (initial droplet). We used the network shown in Fig. 1 and shown schematically in Fig. 2a. Streams $\mathrm{R}_{1}$ and $\mathrm{R}_{2}$ were aqueous solutions of $\mathrm{KNO}_{3}$, stream $\mathrm{S}$ was an aqueous solution of $\mathrm{Fe}(\mathrm{SCN})_{x}(3-x)+$ complexes in $\mathrm{KNO}_{3}$, and stream $\mathrm{R}_{3}$ was an aqueous solution of $\mathrm{KNO}_{3}$. We flowed all four aqueous streams (streams $\mathrm{R}_{1}-\mathrm{R}_{3}$ and $\mathrm{S}$ ) at the same flow rate, and held the water fraction constant. Water fraction, $w f$, is defined as $V_{\text {water }} /\left(V_{\text {water }}+V_{\text {oil }}\right)$ where $V_{\text {water }}[\mathrm{nL}$ $\mathrm{s}^{-1}$ ] is the combined volumetric flow rate of all the aqueous streams, and $V_{\text {oil }}\left[\mathrm{nL} \mathrm{s}^{-1}\right]$ is the volumetric flow rate of the stream of oil.

The behavior of the flow at the merging junction followed the trend illustrated in Fig. 2. At low total flow velocities of $67 \mathrm{~mm} \mathrm{~s}^{-1}$ and below (Fig. 2b), the aqueous stream immediately coalesced with the initial droplet and injected solution into the droplet. At a critical higher flow velocity of $133 \mathrm{~mm} \mathrm{~s}^{-1}$ (Fig. 2c), the aqueous stream $R_{3}$ formed a visible interface with the droplet. The interface did not immediately disappear, but the droplet coalesced with the reagent stream just downstream of the junction. At even higher total flow velocities of $400 \mathrm{~mm} \mathrm{~s}^{-1}$, the aqueous stream $\mathrm{R}_{3}$ formed an interface with the initial droplet, but the interface never broke (Fig. 2d). A separate droplet of the additional reagent was formed, the two droplets became separated by the flow, and no merging took place.

When even higher flow velocities were needed to be accessed for synthesis, we were able to increase the flow velocity at which merging takes place to $400 \mathrm{~mm} \mathrm{~s}^{-1}$ by gently tapping the PDMS microfluidic chip at $10 \mathrm{~Hz}$. This mechanical agitation did not affect the formation of droplets. At the flow velocity of $400 \mathrm{~mm} \mathrm{~s}^{-1}$, initial droplets both formed and merged at a rate of $\sim 600$ droplets per second. We do not fully understand how such a low frequency $(10 \mathrm{~Hz})$ agitation facilitates such a high-frequency $(\sim 600 \mathrm{~Hz})$ coalescence, but it presumably helps break up the interface between an initial droplet and the aqueous reagent stream $R_{3}$.

Coalescence of the aqueous stream with a moving droplet may be thought of in terms of the Capillary number, $\mathrm{Ca}=\mu U / \gamma$, where $\gamma$ $\left[\mathrm{N} \mathrm{m}^{-1}\right]$ is the surface tension at the interface between the aqueous and fluorinated phases. ${ }^{27-31}$ At low values of the Capillary number, surface tension effects dominate and the interface between the stream and the passing droplet quickly dissipates. At high values of the Capillary number, shear forces dominate and oil cannot be displaced from the region between the droplet and aqueous stream as easily. To test this explanation, we first varied the viscosities of the fluids. We performed a similar experiment to the one already described, except the solutions were made more viscous. In the first experiment, both aqueous solutions and oil had the viscosity of water ( $\sim 1 \mathrm{mPa} \mathrm{s})$. We increased the viscosity of both to $\sim 15 \mathrm{mPa}$ $\mathrm{s}$ by adding glycerol to the aqueous solutions, and we used a different fluorinated oil mixture. For fluids with low viscosity, the critical flow velocity (corresponding to Fig. 2c) was $133 \mathrm{~mm} \mathrm{~s}^{-1}$, and for fluids with high viscosity, the critical flow velocity was 7 $\mathrm{mm} \mathrm{s}^{-1}, \sim 15$ times lower. Second, we varied surface tension. In all the previous experiments, we used mixtures of fluorocarbons with $1 \%$ surfactant by weight. We increased the surfactant concentration to $10 \%$ by weight and observed no coalescence, even at slow flow rates of $13 \mathrm{~mm} \mathrm{~s}^{-1}$. The arrest of coalescence by increasing surfactant has previously been observed in a high pressure microfluidic emulsification device. ${ }^{32}$ However, a Capillary number calculated using the surface tension measured at equilibrium probably does not describe this system accurately. When a droplet forms, surface tension at the aqueous/oil interface is greater than that measured at equilibrium because surfactant has not been given time to assemble at the surface of the droplet. As the droplet is transported through the microfluidic channels, more surfactant assembles onto the surface but the surface tension probably does not reach its equilibrium value at the merging junction.

We investigated whether water fraction played a significant role in the behavior at the junction where droplets were injected with additional reagent. We observed that at low water fraction, droplets were small with large separations of oil between them, as observed previously. ${ }^{25}$ One may expect that at low water fractions the additional reagent stream $R_{3}$ would simply form separate droplets in the long segments of oil rather than merge with initial droplets. At a low flow velocity of $67 \mathrm{~mm} \mathrm{~s}^{-1}$ and low water fraction of $w f$ $=0.2$, however, the aqueous stream still injected solution into initial droplets and did not form separate droplets (Fig. 3b). We observed that at high water fractions, initial droplets were long with little oil between, as observed previously. ${ }^{25}$ One may also expect that at high water fractions, the additional reagent stream would always merge with passing droplets. However, at a high flow velocity of $400 \mathrm{~mm} \mathrm{~s}^{-1}$ and high water fraction of $w f=0.8$, the aqueous stream did not join with the droplets despite the fact that there was more time for the interface between the two to dissipate. Instead, the additional reagent stream broke up into separate droplets that segmented the larger, initial droplets (Fig. 3c). We found that the parameters $U, \mu$, and $\gamma$ primarily determine whether the aqueous stream $R_{3}$ will merge with initial droplets or not. Dependence on water fraction is weaker, although we observed that for higher water fraction, the aqueous stream $\mathrm{R}_{3}$ merged more easily with initial droplets for given $U, \mu$, and $\gamma$. These observations show that the Capillary number rather than water fraction played the dominant role in predicting merging of a stream into droplets.

Transport of solids formed by reactions not contained within droplets presented a problem for us when synthesizing colloids using microfluidic channels, even though surfactant stabilized colloidal particles can be handled and assembled in microfluidic devices. Solid reaction products either formed directly on the surface of the channels or sedimented out onto the surface from the reaction mixture, eventually obstructing the flow. For example, when we flowed a stream of $\mathrm{CdCl}_{2}\left(\right.$ as $\mathrm{R}_{1}$ ) and a stream of $\mathrm{Na}_{2} \mathrm{~S}$ (as $R_{2}$ ) together with a stream of buffer (as $S$ ) into a stream of water instead of a stream of oil, we observed that solid CdS built up throughout the channel (Fig. 4a).

We prevented solid reaction products from accumulating on the microchannel walls by confining the reaction within droplets. Previously, colloidal assemblies have been generated in microfluidic channels ${ }^{33,34}$ and water-in-oil droplets have been used to transport assemblies of micron-sized latex beads through channels. ${ }^{35,36}$ We flowed a stream of $\mathrm{CdCl}_{2}\left(\mathrm{R}_{1}\right)$ and a stream of $\mathrm{Na}_{2} \mathrm{~S}$ $\left(R_{2}\right)$ together, separated by a stream of buffer $(S)$, and flowed all 
three of these streams into a stream of fluorinated oil. Negligible buildup was observed at the junction where droplets formed because reagents did not have enough time to diffuse through the separating stream. Once the aqueous reagents and separating aqueous stream formed droplets, a thin layer of fluorinated oil coated the droplets, ${ }^{37,38}$ preventing the CdS product from interacting with the walls of the channel. Fig. $4 \mathrm{~b}$ shows products transported by droplets and no buildup of solids on the walls of channels after a period of $30 \mathrm{~min}$.

Previously, turbulence-based, controlled double-jet precipitation process with rapid mixing has been used for synthesis of colloidal nanoparticles from aqueous precursors in single and multiple step reactions. ${ }^{39}$ Alternatively, turbulence-based mixing of various solvents with supercritical fluid enhanced by an ultrasound field has been used to precipitate protein, ${ }^{40}$ and drug, ${ }^{41}$ nanoparticles of controlled size and distribution. Continuous flow microfluidic methods for synthesis of nanoparticles have also appeared in literature. In these systems, enhanced mixing of reagents ${ }^{42}$ and stream segmentation with nitrogen bubbles ${ }^{43}$ has been shown to increase monodispersity of resulting nanoparticles. Size of resulting nanoparticles was tuned by controlling concentrations of reagents and temperature. ${ }^{44-46}$ An interface between two immiscible, continuously flowing fluids has been used to produce colloidal particles. ${ }^{47}$ The duration of the reaction was defined by the time the reaction mixture spent in a heated region of a microfluidic device; this time was changed by changing the flow velocity through that region. ${ }^{44-46}$ Reaction times as short as $30 \mathrm{~s}$ have been investigated using this method. ${ }^{46}$ Similarly, multi-step core-shell particle synthesis in microchannels has been done with continuous flow systems with tandem hot regions. ${ }^{9}$ This method of controlling reaction times on the order of seconds with temperature is useful for TOPO based synthesis of nanoparticles, ${ }^{48}$ but is less useful for faster multistep reactions on millisecond time scale. We have been able to combine many of these ideas with a droplet-based microfluidic platform to allow for multi-step synthesis on millisecond timescale.

We demonstrated the utility of this microfluidic method by using it to synthesize $\mathrm{CdS}$ nanoparticles from aqueous precursors at room temperature. In the first experiment, a mixture of $\mathrm{CdCl}_{2}$ and mercaptopropionic acid (MPA) was infused through the left aqueous inlet of the microfluidic device (Fig. 5a). A solution of $\mathrm{Na}_{2} \mathrm{~S}$ was infused through the right aqueous inlet. A solution of $\mathrm{NaOH}$ was infused through the middle aqueous inlet to separate the two reactant streams and adjust the $\mathrm{pH}$ of the reaction mixture to $\mathrm{pH}$ 11. These aqueous streams were infused into a flow of oil, forming droplets. Complete mixing in droplets was achieved in less then 5 $\mathrm{ms},{ }^{1}$ initiating the reaction. The droplets were then allowed to react for $75 \mathrm{~ms}$ as they passed through the serpentine section of the device (Fig. 5a). When droplets reached the merging junction they coalesced with the quenching stream containing MPA (labeled "QUENCH" in Fig. 5a). Mixing of the reagents within the resulting droplet occurred within $5 \mathrm{~ms}$. It has been shown that MPA forms a protective layer around the nanoparticle and prevents aggregation, ${ }^{49,50}$ thus quenching the reaction. Control on this time-scale gave CdS nanoparticles with a sharp absorption peak at $\lambda_{\max }=325$ $\mathrm{nm}$ (Fig. 5b, curve A). In the second experiment, we performed the reaction under identical conditions as described for the first experiment above, but we did not flow a stream of MPA quenching agent through the quenching inlet. Nanoparticles collected in this experiment had a broader absorption peak indicating a decrease in monodispersity. (Fig. 5b, curve B). For comparison, we then
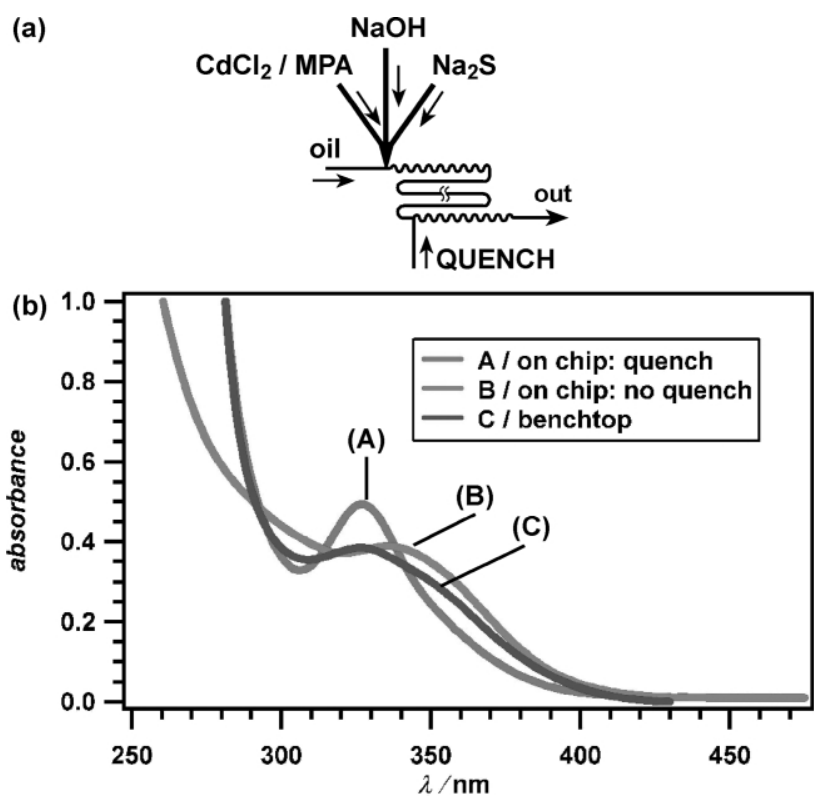

Fig. 5 Two-step synthesis on-chip with millisecond quenching yields CdS colloidal nanoparticles that are less disperse than those synthesized without millisecond quenching. (a) A schematic diagram of the microfluidic network. (b) UV/Vis spectra of nanoparticles synthesized on-chip with millisecond quench (A), on-chip without quench (B), and on the bench top (C).

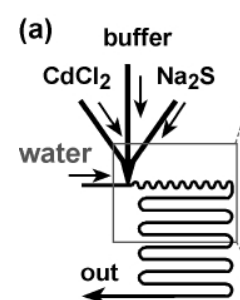

(b)

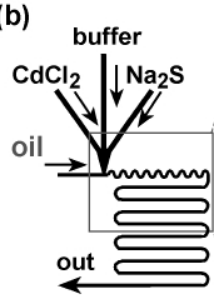

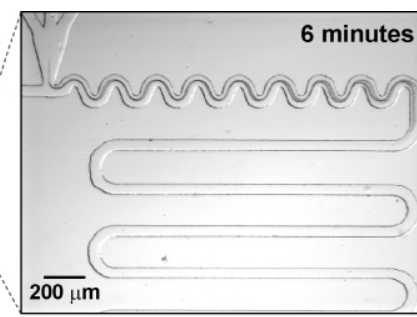
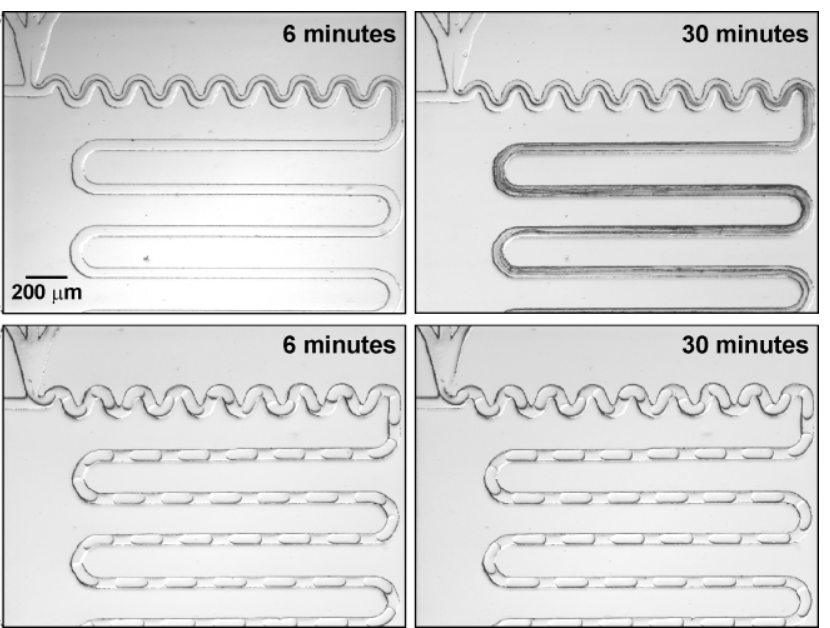

Fig. 4 Reactions that produce solid CdS can be performed in droplets. (a) Left: a schematic diagram of the microfluidic network. Middle: A micrograph showing accumulation of solid CdS on the walls of the channels after 6 min. No oil was used, and reagents were not confined within droplets. Right: A micrograph showing accumulation of solid CdS on the walls of the channels after 30 min. (b) Left: a schematic diagram of the microfluidic network. Middle: A micrograph showing no accumulation of solid CdS on the walls of the channels after 6 min of flow. Oil was used to confine reagents within droplets, preventing products from interacting with the walls of the channels. Right: A micrograph showing no accumulation of solid CdS on the walls after 30 min using droplets. In all images, $U=160 \mathrm{~mm} \mathrm{~s}^{-1}$. Droplets were formed at $w f=0.5$. 
performed a third experiment without microfluidic channels. The same solutions and quenching agent as in the first experiment $\left(\mathrm{CdCl}_{2} / \mathrm{MPA}, \mathrm{NaOH}, \mathrm{Na}_{2} \mathrm{~S}\right.$, and MPA) were pipetted together under vigorous mixing (as described in the experimental section). Again, noticeably less monodisperse $\mathrm{CdS}$ nanoparticles were obtained (Fig. 5b, curve C). These results demonstrate that rapid mixing and quenching on millisecond time scale is essential to produce monodisperse $\mathrm{CdS}$ nanoparticles from these aqueous reagents.

We further demonstrated the utility of this droplet-based microfluidic method by performing four experiments to synthesize $\mathrm{CdS}$ nanoparticles of three sizes and $\mathrm{CdS} / \mathrm{CdSe}$ core-shell particles. In all experiments aqueous $\mathrm{CdCl}_{2} / \mathrm{MPA}$ and $\mathrm{Na}_{2} \mathrm{~S}$ reagents (at various molar ratios) separated by $\mathrm{NaOH}$ solution, were infused into the microfluidic channels (Fig. 6a). These aqueous reagents were allowed to react for $75 \mathrm{~ms}$ and then quenched with either MPA, $\mathrm{Na}_{2} \mathrm{~S}$, or $\mathrm{Na}_{2} \mathrm{Se}$. In the first experiment, 20 fold excess of $\mathrm{CdCl}_{2} / \mathrm{MPA}$ over $\mathrm{Na}_{2} \mathrm{~S}$ was used in the reaction. A stream of MPA was infused through the quench inlet and injected into reaction droplets. This resulted in smaller CdS nanoparticles with absorption shoulder at $\lambda \approx 290 \mathrm{~nm}$ on the thiol absorption edge (Fig. 6b, curve A). We confirmed the presence of CdS nanoparticles by observing fluorescence at $\lambda_{\mathrm{em}}=360 \mathrm{~nm}$ with $\lambda_{\mathrm{ex}}=290 \mathrm{~nm}$. We performed the second experiment with equimolar amounts of $\mathrm{CdCl}_{2} / \mathrm{MPA}$ and $\mathrm{Na}_{2} \mathrm{~S}$. A stream of MPA was infused through the quench inlet and injected into reaction droplets. Larger $\mathrm{CdS}$ nanoparticles were obtained (Fig. 6b, curve B) with absorption $\lambda_{\max }$ $=325 \mathrm{~nm}$ (Same as Fig. 5b, curve A). We performed the third experiment with equimolar amounts of $\mathrm{CdCl}_{2} / \mathrm{MPA}$ and $\mathrm{Na}_{2} \mathrm{~S}$. A stream of $\mathrm{Na}_{2} \mathrm{~S}$ was infused through the quench inlet and injected into droplets of the initial reaction mixture. This reaction gave $\mathrm{CdS}$ nanoparticles with absorption $\lambda_{\max }=370 \mathrm{~nm}$ (Fig. 6b, curve C). Presumably, the observed red shift in absorption maximum is due to the formation of larger nanoparticles by an addition of a sulfiderich outer shell. We performed the fourth experiment also with equimolar amounts of $\mathrm{CdCl}_{2} / \mathrm{MPA}$ and $\mathrm{Na}_{2} \mathrm{~S}$. In contrast to the previous three experiments, a stream of $\mathrm{Na}_{2} \mathrm{Se}$ was infused through the quench inlet and injected into reaction droplets. The resulting reaction gave $\mathrm{CdS} / \mathrm{CdSe}$ core-shell nanoparticles (Fig. 6b, curve
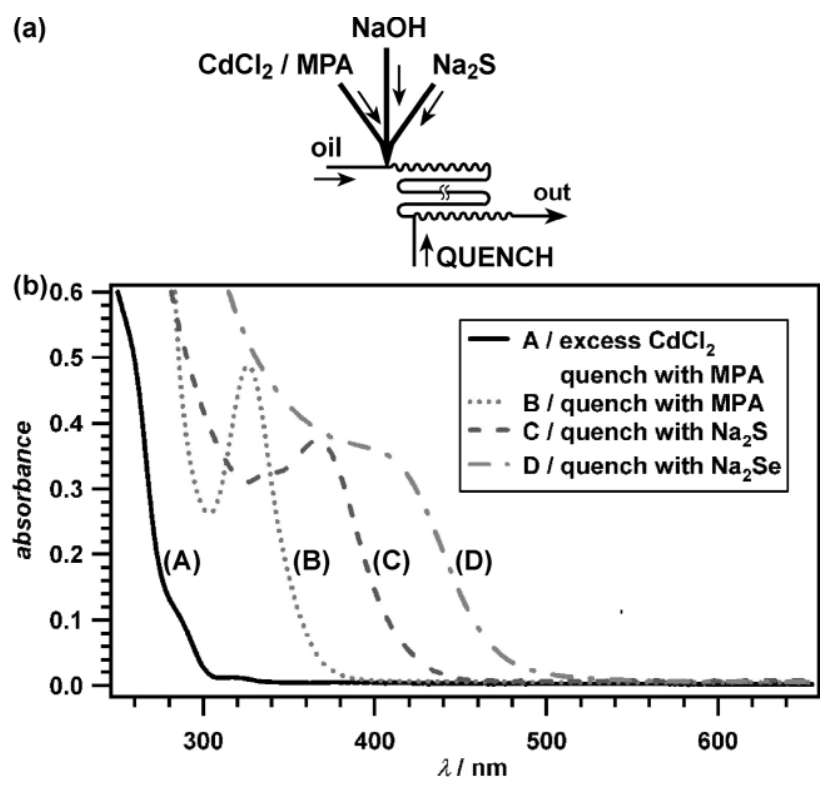

Fig. 6 Two-step synthesis of nanoparticles with various sizes and composition. (a) A schematic diagram of the microfluidic network. (b) UV/ Vis spectra of 4 different types of nanoparticles. (A): CdS nanoparticles synthesized using 20:1 ratio of $\mathrm{CdCl}_{2}$ to $\mathrm{Na}_{2} \mathrm{~S}$ with thiol quench. (B): $\mathrm{CdS}$ nanoparticles synthesized using $1: 1$ ratio of $\mathrm{CdCl}_{2}$ to $\mathrm{Na}_{2} \mathrm{~S}$ with thiol quench. (C): CdS nanoparticles synthesized using $1: 1$ ratio of $\mathrm{CdCl}_{2}$ to $\mathrm{Na}_{2} \mathrm{~S}$ with $\mathrm{Na}_{2} \mathrm{~S}$ quench. (D): $\mathrm{CdS} / \mathrm{CdSe}$ core-shell nanoparticles synthesized using 1:1 ratio of $\mathrm{CdCl}_{2}$ to $\mathrm{Na}_{2} \mathrm{~S}$ with $\mathrm{Na}_{2} \mathrm{Se}$ quench.
D). Such composite nanoparticles have previously appeared in literature. ${ }^{51} \mathrm{We}$ confirmed that the red shift in absorption was due to an addition of a selenium outer shell by observing complete quenching of fluorescence, as expected. ${ }^{52}$ These four experiments illustrate the flexibility of the microfluidic network in accommodating different two-step reaction schemes without changing the geometry of the microfluidic channels.

\section{Conclusion}

We have characterized coalescence of an aqueous stream with moving droplets as a function of the Capillary number, and used this knowledge to perform two-step chemical synthesis of $\mathrm{CdS}$ nanoparticle with millisecond reaction times. As the example, here we used synthesis of $\mathrm{CdS}$ nanoparticles because their formation occurs on millisecond time scale and has been studied previously, and because the reagents for aqueous CdS synthesis can be handled safely. We did not characterize these nanoparticles in detail because it is known that $\mathrm{CdS}$ particles synthesized at room temperature in aqueous solutions are not very crystalline. Extending the dropletbased microfluidic method described in this paper to reagents in non-aqueous solutions and to reactions performed at higher temperatures would lead to synthesis of high-quality nanoparticles by multistep reactions with millisecond time control of nucleation and growth processes. This microfluidic system may also become useful for synthesis of nano-size drug particles with ${ }^{41}$ or without encapsulating layers, and other particles used in pharmaceutical, food, and agricultural industries. Rapid sub-millisecond coalescence required for this system is affected by several parameters that remain to be investigated further: dynamics of self-assembly of surfactant at the aqueous-fluorinated interface, effects of solutions of contrasting viscosities, and effect of mechanical agitation. Once the effect of these parameters is understood, this microfluidic system will be useful for a range of multistep synthetic and analytical reactions taking place on millisecond time scale.

\section{Acknowledgements}

This work was supported by the Beckman Young Investigator Program and Chicago MRSEC funded by NSF. At The University of Chicago, work was performed at the MRSEC microfluidic facility. JDT is a Beckman Scholar. We thank David Sharoyan and Bo Zheng for helpful discussions and preliminary experiments. We thank Cory J. Gerdts and Helen Song for performing photolithography at MAL of the UIC.

\section{References}

1 H. Song, J. D. Tice and R. F. Ismagilov, Angew. Chem. Int. Ed., 2003, 42, 768 .

2 K. F. Jensen, Chem. Eng. Sci., 2001, 56, 293.

3 H. A. Stone, A. D. Stroock and A. Ajdari, Annu. Rev. Fluid Mech., 2004, 36, 381

4 H. A. Stone and S. Kim, Aiche J., 2001, 47, 1250

5 R. F. Ismagilov, Angew. Chem. Int. Edit., 2003, 42, 4130.

6 T. Thorsen, S. J. Maerkl and S. R. Quake, Science, 2002, 298, 580.

7 G. H. Seong and R. M. Crooks, J. Am. Chem. Soc., 2002, 124, 13360.

8 P. D. I. Fletcher, S. J. Haswell, E. Pombo-Villar, B. H. Warrington, P Watts, S. Y. F. Wong and X. L. Zhang, Tetrahedron, 2002, 58, 4735

9 H. Z. Wang, X. Y. Li, M. Uehara, Y. Yamaguchi, H. Nakamura, M. P. Miyazaki, H. Shimizu and H. Maeda, Chem. Commun., 2004, 48.

10 M. A. Burns, B. N. Johnson, S. N. Brahmasandra, K. Handique, J. R. Webster, M. Krishnan, T. S. Sammarco, P. M. Man, D. Jones, D. Heldsinger, C. H. Mastrangelo and D. T. Burke, Science, 1998, 282, 484.

11 J. R. Burns and C. Ramshaw, Chem. Eng. Commun., 2002, 189, 1611.

12 N. de Mas, A. Gunther, M. A. Schmidt and K. F. Jensen, Ind. Eng. Chem. Res., 2003, 42, 698. 
13 M. W. Losey, R. J. Jackman, S. L. Firebaugh, M. A. Schmidt and K. F. Jensen, J. Microelectromech. Syst., 2002, 11, 709.

14 J. R. Burns and C. Ramshaw, Lab Chip, 2001, 1, 10.

15 R. F. Ismagilov, A. D. Stroock, P. J. A. Kenis, G. Whitesides and H. A. Stone, Appl. Phys. Lett., 2000, 76, 2376.

16 R. B. Bird, W. E. Stewart and E. N. Lightfoot, Transport Phenomena, Wiley, New York, 2002.

17 A. E. Kamholz and P. Yager, Sens. Actuators, B, 2002, 82, 117

18 M. C. R. Shastry, S. D. Luck and H. Roder, Biophys. J., 1998, 74, 2714.

19 H. Song, M. R. Bringer, J. D. Tice, C. J. Gerdts and R. F. Ismagilov, Appl. Phys. Lett., 2003, 83, 4664.

20 H. Song and R. F. Ismagilov, J. Am. Chem. Soc., 2003, 125, 14613.

21 B. Zheng, L. S. Roach and R. F. Ismagilov, J. Am. Chem. Soc., 2003, 125, 11170.

22 B. Zheng, J. D. Tice, S. L. Roach and R. F. Ismagilov, Angew. Chem. Int. Ed., 2004, 43, 2508.

23 S. L. Anna, N. Bontoux and H. A. Stone, Appl. Phys. Lett., 2003, 82, 364.

24 T. Thorsen, R. W. Roberts, F. H. Arnold and S. R. Quake, Phys. Rev. Lett., 2001, 86, 4163.

25 J. D. Tice, H. Song, A. D. Lyon and R. F. Ismagilov, Langmuir, 2003 , 19, 9127.

26 A. Gunther, M. Jhunjhunwala, M. A. Schmidt and K. F. Jensen, Proc. MicroTAS, 2003, 1, 465.

27 H. Yang, C. C. Park, Y. T. Hu and L. G. Leal, Phys. Fluids., 2001, 13, 1087.

28 S. D. Hudson, A. M. Jamieson and B. E. Burkhart, J. Colloid Interface Sci., 2003, 265, 409.

29 Y. T. Hu, D. J. Pine and L. G. Leal, Phys. Fluids, 2000, 12, 484.

30 M. A. Rother and R. H. Davis, Phys. Fluids, 2001, 13, 1178.

31 J. A. Pathak and K. B. Migler, Langmuir, 2003, 19, 8667.

32 L. Lobo and A. Svereika, J. Colloid Interface Sci., 2003, 261, 498.

33 E. Kumacheva, P. Garstecki, H. K. Wu and G. M. Whitesides, Phys. Rev. Lett., 2003, 91.
34 D. S. W. Lim, J. P. Shelby, J. S. Kuo and D. T. Chiu, Appl. Phys. Lett., 2003, 83, 1145.

35 G. R. Yi, T. Thorsen, V. N. Manoharan, M. J. Hwang, S. J. Jeon, D. J. Pine, S. R. Quake and S. M. Yang, Adv. Mater., 2003, 15, 1300.

36 G. R. Yi, S. J. Jeon, T. Thorsen, V. N. Manoharan, S. R. Quake, D. J. Pine and S. M. Yang, Synth. Met., 2003, 139, 803.

37 J. Bico and D. Quéré, J. Fluid Mech., 2002, 467, 101.

38 J. Bico and D. Quéré, J. Colloid Interface Sci., 2002, 247, 162.

39 I. Sondi, O. Siiman, S. Koester and E. Matijevic, Langmuir, 2000, 16, 3107.

40 P. Chattopadhyay and R. B. Gupta, Aiche J., 2002, 48, 235.

41 P. Chattopadhyay and R. B. Gupta, Ind. Eng. Chem. Res., 2002, 41, 6049.

42 J. B. Edel, R. Fortt, J. C. deMello and A. J. deMello, Chem. Commun., $2002,1136$.

43 H. Nakamura, Y. Yamaguchi, M. Miyazaki, H. Maeda, M. Uehara and P. Mulvaney, Chem. Commun., 2002, 2844.

44 E. M. Chan, R. A. Mathies and A. P. Alivisatos, Nano Lett., 2003, 3, 199.

45 B. K. H. Yen, N. E. Stott, K. F. Jensen and M. G. Bawendi, Adv. Mat., 2003, 15, 1858

456 H. Nakamura, Y. Yamaguchi, M. Miyazaki, M. Uehara, H. Maeda and P. Mulvaney, Chem. Lett., 2002, 1072.

47 H. Z. Wang, H. Nakamura, M. Uehara, M. Miyazaki and H. Maeda, Chem. Commun., 2002, 1462

48 C. B. Murray, D. J. Norris and M. G. Bawendi, J. Am. Chem. Soc., 1993, 115, 8706.

49 L. Wang, L. Y. Wang, C. Q. Zhu, X. W. Wei and X. W. Kan, Anal. Chim. Acta, 2002, 468, 35.

50 D. Lawless, S. Kapoor and D. Meisel, J. Phys. Chem., 1995, 99, 10329.

51 Y. Xie, P. Yan, J. Lu, Y. Qian and S. Zhang, Chem. Commun., 1999, 1969.

52 Y. Tian, T. Newton, N. A. Kotov, D. M. Guldi and J. H. Fendler, J. Phys. Chem., 1996, 100, 8927. 